\title{
Optical Rotation Detection for Atomic Spin Precession Using a Superluminescent Diode
}

\author{
Xuejing LIU, Yang LI, Hongwei CAI, Ming DING ${ }^{*}$, Jiancheng FANG, and Wei JIN \\ School of Instrumentation Science and Opto-electronics Engineering, Beihang University, Beijing 100191, China \\ *Corresponding author: Ming DING_E-mail: mingding@buaa.edu.cn
}

\begin{abstract}
A superluminescent diode (SLD) as an alternative of laser is used to detect optical rotation for atomic spin precession. A more uniform Gauss configuration without additional beam shaping and a relatively high power of the SLD have a potential for atomic magnetometers, which is demonstrated in theory and experiments. In addition, the robustness and compactness enable a more practical way for optical rotation detections, especially for applications in magnetoencephalography systems.
\end{abstract}

Keywords: Superluminescent diode; atomic magnetometer; magnetoencephalography; atomic spin precession detection; Larmor precession

Citation: Xuejing LIU, Yang LI, Hongwei CAI, Ming DING, Jiancheng FANG, and Wei JIN, "Optical Rotation Detection for Atomic Spin Precession Using a Superluminescent Diode," Photonic Sensors, 2019, 9(2): 135-141.

\section{Introduction}

Precise probing of ensemble of atoms with light waves has been central to exploit the magnetic properties of atomic ensemble to perform precise magnetometry [1]. In fact, the atomic magnetometers (AMs) have reached a sensitivity of sub-femtotesla [2] and offer an alternative in ultra-high sensitivity magnetometers previously dominated by superconducting quantum interference devices (SQUIDs) [3]. Such high sensitivity can be achieved directly at room temperature without any cryogenic requirements and associated complex logistics [4]. As a result, the atomic magnetometer has a compact structure, providing a new solution with better spatial resolutions for magnetoencephalography [5] and magnetocardiography systems [6]. On the other hand, the operation of atomic magnetometers is much simpler than that of SQUID magnetometers, allowing free and natural movement for patients during the scanning process [7-9]. Moreover, the atomic magnetometer has a bandwidth ranging from static to hundreds of kilohertz [10], which can be applied to more fields such as detection of microparticle in size of micrometers [11] and magnetic particle imaging (MPI) [12].

In atomic magnetometers, a glass cell containing alkali atomic vapor is illuminated with pump light that is resonant with electric transitions. The atoms generate precession when exposed to magnetic fields. The precession signal could be detected using absorption spectroscopy [13] or by a probe light with the off-resonant linear polarization state [14]. For the magnetoencephalography (MEG) system, the sensor is a helmet covering the head containing an array of magnetometers [15], which means that hundreds of headers are installed within a small

Received: 25 October 2018 / Revised: 18 January 2019

(C) The Author(s) 2019. This article is published with open access at Springerlink.com

DOI: $10.1007 / \mathrm{s} 13320-019-0539-8$

Article type: Regular 
space about $100 \mathrm{~cm}^{3}$ [16]. Such requirement is critical to both pump and probe lights, as the laser with the asymmetry of the divergence angle needs spatial beam shaping, which can improve the measurement precision while induces the complication and wrecks the compactness. In this case, the laser source could be separated from the sensor header [17] via a polarization maintaining fiber (PMF). However, the light intensity drifts with the use of the PMF as the laser is a polarized light source [18]. On the other hand, some portable device chooses a vertical cavity surface emitting (VCSEL) laser for its advantages of compactness and robustness. However, the pump and probe lights need polarized lights, while VCSEL laser has instability of polarization states with the results of its specific structure [19]. Thus, some other light sources may also be considered, such as the alkali discharge lamp, which is used for atomic spectroscopy years early before the lasers [20]. Although the wavelength is no doubt suitable, the coupling efficiency to fiber is the key limitation as it radiates around with limited light intensity [21].

The superluminescent diode (SLD) has been widely used for many applications such as optical gyroscopes, optical time-domain reflectometers (OTDRs), optical coherence tomography (OCT) systems, and wavelength division-multiplexing (WDM) testing systems [22]. For these applications, the SLD is of great interest as light sources owing to its robustness, compactness, cost-effectiveness, and better spatial characteristics [23-25]. Compared with the traditional alkali discharge lamps, the SLD is a non-polarizing light source and also has better coupling efficiency to the fiber. In this paper, an SLD as a probe light is used in the spin-exchange relaxation free (SERF) atomic magnetometer. The theoretical and experimental analysis demonstrates that the SLD has a potential for interrogating the optical rotation for atomic spin precession.

\section{Principle}

Figure 1 shows the principles of the SERF atomic magnetometer. A circular polarized pump laser centered at the atomic resonance wavelength polarizes the alkali atoms. Polarized atoms generate precession in the magnetic fields. When an off-resonance probe beam with a linear polarization state passes through atoms, the polarization of the probe beam rotates with an angle of $\theta$ linear to the magnetic field $B_{y}$ in the $y$ direction, which is described as [26]

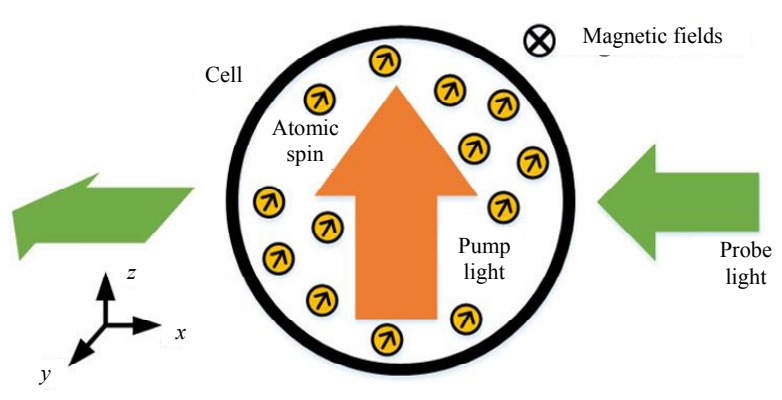

Fig. 1 Principle of SERF atomic magnetometer.

$$
\begin{aligned}
\theta= & \frac{1}{2} l r_{e} n c f_{D 1}\left[-D_{D 1}\left(v-v_{D 1}\right)+2 D_{D 2}\left(v-v_{D 2}\right)\right] . \\
& P_{0} \frac{\gamma_{e} R_{\mathrm{tot}}}{R_{\mathrm{tot}}^{2}+\left(\gamma_{e} B_{y}\right)^{2}} B_{y}
\end{aligned}
$$

where $l$ is the length of the vapor cell, $r_{e}$ is the classical electron radius, $n$ is the density of the alkali vapor, and $c$ is the light speed. $f_{D 1} \approx 1 / 3$ is the oscillator strength of given atomic resonance.

$$
\begin{gathered}
D_{D i}\left(v-v_{D i}\right)=\left(v-v_{D i}\right) /\left[\left(v-v_{D i}\right)^{2}+(\Delta v / 2)^{2}\right] \\
(i=1,2)
\end{gathered}
$$

is the atomic frequency response with a dispersion shape. $v$ is the frequency of the probe beam, $v_{D 1}$ and $v_{D 2}$ are the resonance frequencies corresponding to the D1 and D2 transitions of alkali atoms respectively, $P_{0}$ is the atomic spin polarization, $\gamma_{e}$ is the gyromagnetic ratio, $R_{\mathrm{tot}}$ is the total relaxation of atoms, and $B_{y}$ is the magnetic field in the $y$ direction.

The overall atomic spin precession signal $S$ is determined by both the optical rotation $\theta$ and 
beam absorption [27], which is

$$
S=I_{0} \theta \mathrm{e}^{-O D}
$$

where $I_{0}$ is the incident intensity of probe laser on the atomic vapor, and $O D$ is the optical absorption coefficient of alkali atoms with a pressure broadening factor $\Gamma$ as follows [28]:

$$
O D=n l \pi r_{e} c f \frac{\Gamma / 2}{\left(v-v_{0}\right)^{2}+(\Gamma / 2)^{2}} .
$$

The vapor cell in the present experiment has a spherical shape with a diameter of $2 \mathrm{~cm}$, containing potassium with the density of $5 \times 10^{13} \mathrm{~cm}^{-3}, 60$ Torr $\mathrm{N}_{2}$ and $3 \mathrm{~atm}$ He. The pump and probe beams are with $10 \mathrm{~mm}$ and $5 \mathrm{~mm}$ diameter respectively, and they propagate perpendicularly in free space.

Considering the SLD with the frequency from $v_{1}$ to $v_{2}$, the optical rotation is modified to be the sum of the optical rotation at each frequency according to (1), which is as follows:

$$
\begin{gathered}
\theta=\int_{v 1}^{v 2} \frac{1}{2} l r_{e} c f_{D 1}\left[-D_{D 1}\left(v_{i}\right)+D_{D 2}\left(v_{i}\right)\right] . \\
\frac{1}{2} P_{0} \frac{\gamma_{e} R_{\mathrm{tot}}}{R_{\mathrm{tot}}^{2}+\left(\gamma_{e} B_{y}\right)^{2}} B_{y} d v_{i} .
\end{gathered}
$$

The intensity of the SLD with the function of the frequency is defined as $I\left(v_{i}\right)$, and the atomic spin precession signal is modified as follws:

$$
\begin{gathered}
S=\int_{v 1}^{v 2} \frac{I\left(v_{i}\right)}{2} l r_{e} c f_{D 1}\left[-D_{D 1}\left(v_{i}\right)+D_{D 2}\left(v_{i}\right)\right] . \\
P_{0} \frac{\gamma_{e} R_{\mathrm{tot}}}{R_{\mathrm{tot}}^{2}+\left(\gamma_{e} B_{y}\right)^{2}} B_{y} d v_{i} .
\end{gathered}
$$

Figure 2 shows the simulated spectra of the SLD in the black line and the atomic spin precession signal in the red line respectively according to (1) and (2). In simulations, the power of the SLD is $20 \mathrm{~mW}$, which is the common performance for a commercial SLD. The wavelength of the SLD is chosen between D1 and D2 lines of rubidium. As the regular SLD has a $4.84 \mathrm{THz}-9.68 \mathrm{THz}$ linewidth, the frequency range is chosen from $377.4 \mathrm{THz}$ to $384.6 \mathrm{THz}$, where both amplitudes of light intensity and precession signal are strong. The red line is the normalized signal of atomic precession with the
SLD described above. Although the single signal at a certain wavelength is small compared with the laser source, the integral signals are $1.57 \times 10^{-7}$ Arb. units. In the same situation, the signal amplitude with a laser source is also simulated. The wavelength of the laser is centered at $377.4 \mathrm{~Hz}$, the power is $6 \mathrm{~mW}$, and the linewidth is $10 \mathrm{MHz}$. Using (1) and (2), the max signal with the laser is $1.42 \times 10^{-7}$ Arb. units, which is in the same level with that of the SLD system. These results prove that the SLD has the same potential for the atomic spin precession detection compared with the laser source. However, the SLD described above needs to be custom-made, and a standard commercial SLD (SLD-331, Superlum) is used in the experiments as follows.

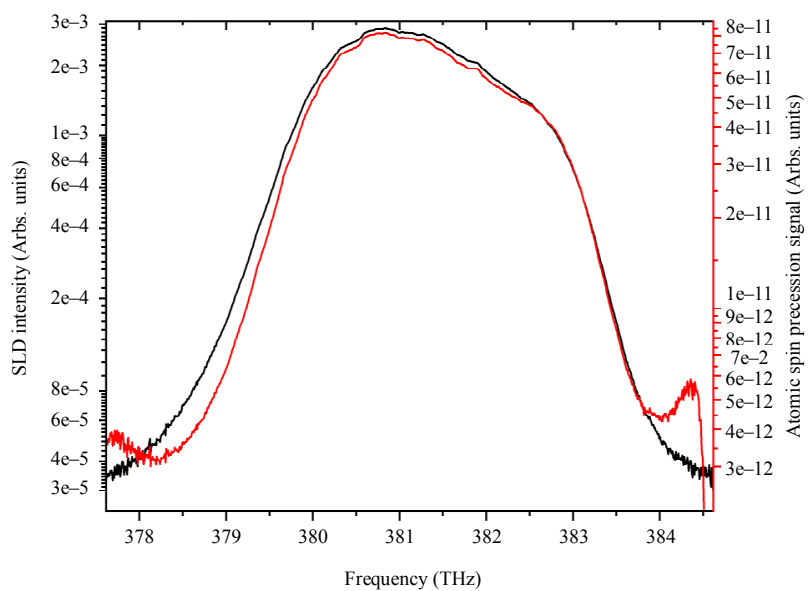

Fig. 2 Spectra of the SLD and the atomic spin precession signal with the SLD.

\section{Experimental setup and results discussion}

The feasibility of the SLD for atomic spin precession detection is verified with K-Rb SERF atomic magnetometer shown in Fig. 3. The atomic vapor cell is placed inside a 4-layer $\mu$-metal magnetic shielding. The shields provide the geomagnetic fields attenuation with the magnitude of $10^{6}$. Magnetic coils mounted inside the shield are used for compensation of residual magnetic fields and produce a calibration magnetic field along the $y$ 
axis. In order to optimize the operation of the atomic magnetometer, the residual magnetic field inside the shields with a level of $2 \mathrm{nT}$ should be zeroed. All three magnetic field components are zeroed with tri-axial coils according to a cross-modulation technique to within $10 \mathrm{pT}$ [29].

A sample of $\mathrm{K}$ and $\mathrm{Rb}$ contained in a 2-cm-diameter spherical glass cell is with 2.5 atm of ${ }^{4} \mathrm{He}$ and 60 Torr nitrogen $\left(\mathrm{N}_{2}\right)$. The atomic number density ratio is about $R=\mathrm{K} / \mathrm{Rb}=1 / 180$. The $\mathrm{N}_{2}$ is used as the quenching gas to eliminate radiation trapping and improve optical pumping efficiency, while ${ }^{4} \mathrm{He}$ is the buffer gas to reduce the diffusion rate of atoms from the central part to the cell walls.

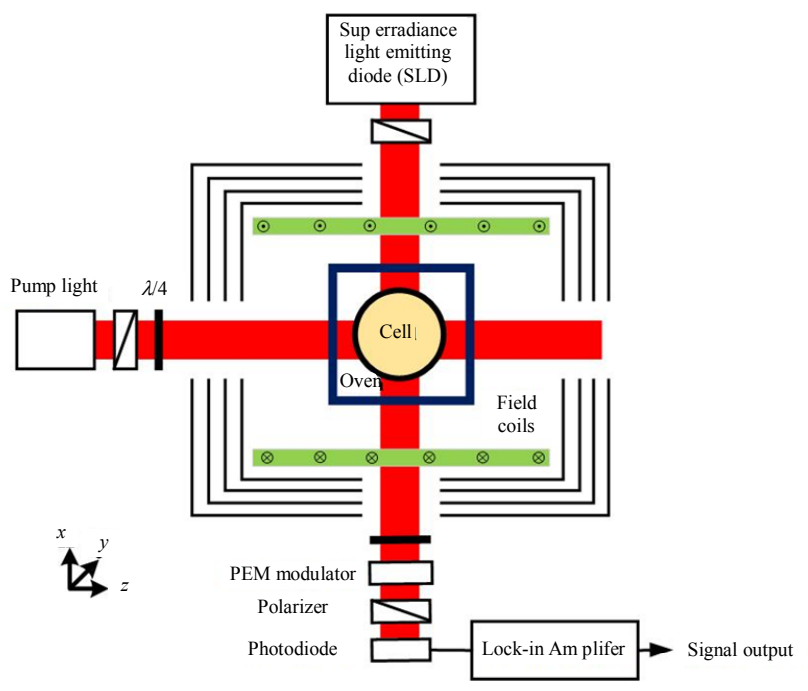

Fig. 3 Schematic of the atomic magnetometer.

The vertical pump and probe lights interact in the vapor cell and propagate along $z$ and $x$ directions. The external cavity diode laser (model 6910, New Focus) produces the pump beam with $15 \mathrm{~mW}$ at $770 \mathrm{~nm}$ for the potassium D1 line. The laser is circularly polarized by passing through a polarizer and quarter wave plate with a cross angle of $45^{\circ}$ between optical axes.

An SLD (SLD-331, Superlum) produces a probe beam with the power of $22 \mathrm{~mW}$ and wavelength from $753 \mathrm{~nm}$ to $855 \mathrm{~nm}$ (from $350.9 \mathrm{THz}$ to $398.4 \mathrm{THz}$ ) which covers the D1 and D2 lines of rubidium. The probe beam passes through a polarizer to define initial polarization state of the probe beam. When the SERF magnetometer feels the magnetic field, the polarization of the probe beam changes. A photoelastic modulator (PEM) modulator is followed after a reflector to modulate probe beam polarization with an amplitude of $0.08 \mathrm{rad}$ at $5 \mathrm{kHz}$ [30]. After the shielding, the polarization of the probe beam is analyzed with a polarizer perpendicular to the initial polarization of the probe beam. The output signal is obtained by entering the probe beam onto a photodiode. The atomic spin precession signal is extracted from the first harmonic of the PEM modulation frequency with a lock-in amplifier (model HF2LI, Zurich Instrument, Switzerland).

Figure 4 shows the response for the $\mathrm{K}-\mathrm{Rb}$ atomic magnetometer with the SLD to a $66.5 \mathrm{pT}$ at $30 \mathrm{~Hz}$ calibrated magnetic field signal. The signal is recorded by a data acquisition (DAQ) system with the sample rate of $1000 / \mathrm{s}$ and sampling time of $100 \mathrm{~s}$. The frequency spectrum is obtained using the fast Fourier transition. The peak at $30 \mathrm{~Hz}$ is the atomic spin precession signal, as it disappears along with the calibrated magnetic fields. Other peaks, such as that at $50 \mathrm{~Hz}$, independent with the calibrated signal prove that they are noise peaks and industrial signal. The amplitude of the peak at $30 \mathrm{~Hz}$ represents the scale factor of the system. The noise level at $30 \mathrm{~Hz}$ represents the limited sensitivity of the system, which is estimated to be $54.6 \mathrm{fT} \cdot \mathrm{Hz}^{-1 / 2}$ at $30 \mathrm{~Hz}$ according to the scale factor measured. The results show the possibility of applications on biomedical fields using the SLD system, as the magnetic fields generated by human brain is less than $100 \mathrm{fT}$ [4]. Moreover, the frequency of the SLD used in experiments ranges from $357.14 \mathrm{THz}$ to $400 \mathrm{THz}$, which is much broader than that of the laser with the same power. The power of the SLD used in the experiment is over 3 times than that of the laser, and the linewidth is 241.3 times wider, which means that the light density of the SLD is only $1.7 \%$ that of the 
laser. If the SLD described in the simulation sections is used, the signal could be improved by 90.3 times, which is equivalent to $0.6 \mathrm{fT} / \mathrm{Hz}^{1 / 2}$ in ideal conditions.

Figure 5 shows responses to different calibrated magnetic fields with the SLD at $22 \mathrm{~mW}$ and laser at $6 \mathrm{~mW}$ experimentally. The signals are both linear to the calibration magnetic fields, and their amplitude ratio is 27.1. The experimental results are close to the simulation results using (1) and (2), which is 27.5 theoretically. The coincident results verify that the theoretical analyses are reasonable, and the method we proposed is feasible.

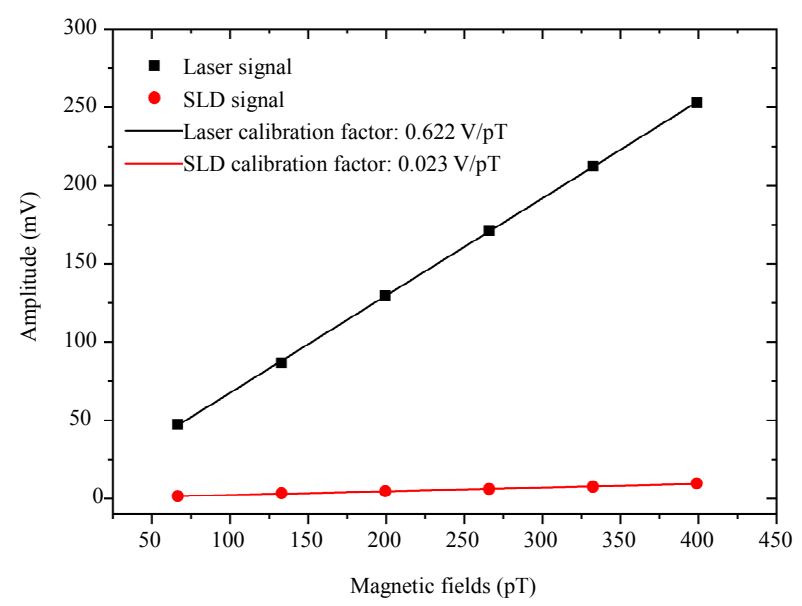

Fig. 5 Response for different magnetic fields of K-Rb SERF magnetometers in the $y$ directions with the SLD and laser source, respectively. The dots and line in black represent the results with laser source, and those in red are the results with the SLD.

By optimizing the linewidth and the power of the SLD, the signal of the atomic spin precession detection with the SLD could be improved. In addition, the SLD is a non-polarizing light source with better choice in the application of the MEG system for matching up the fiber operation. Moreover, the SLD could be adapted for more complicated environment with the simpler and smaller structure, which has a potential for atomic spin precession detection in the application of the MEG systems and opens up more possibility as a new light source for spin precession detection in other fields, such as MPI, microparticle detection.

\section{Conclusions}

A SERF atomic magnetometer with an SLD as a probe light source is proposed. The theoretical and experimental analysis demonstrates the sensitivity compared with the laser source. The sensitivity of the atomic magnetometer with $\mathrm{K}-\mathrm{Rb}$ atoms using an SLD as the probe light source is evaluated to be $54.6 \mathrm{fT} \cdot \mathrm{Hz}^{-1 / 2}$ at $30 \mathrm{~Hz}$, which proves that the SLD could be a new probe light source for SERF atomic magnetometers. The atomic spin precession signal in experiment agrees with that of theoretical analysis, which can be improved by optimizing the linewidth and power intensity of the SLD. In addition, as the SLD is a non-polarizing light source, the atomic magnetometer using the SLD has a better stability compared with the laser source when fibers are delivering light to senor headers. Moreover, the compactness, robustness, and cost-effectiveness of the SLD has a better adaption for complicated environment for different applications. The SLD opens up a new way for atomic spin precession detection, with applications such as the SERF atomic magnetometer, and array detectors of MEG systems, MPI system, and microparticle detection.

\section{Acknowledgment}

This work is supported by the National Key Research and Development Program of China under Grant Nos. 2017YFB0503100 and 2016YFB051600.

Open Access This article is distributed under the terms of the Creative Commons Attribution 4.0 International License (http://creativecommons.org/licenses/by/4.0/), which permits unrestricted use, distribution, and reproduction in any medium, provided you give appropriate credit to the original author(s) and the source, provide a link to the Creative Commons license, and indicate if changes were made.

\section{References}

[1] W. Happer and B. S. Mathur, "Off-resonant light as a probe of optically pumped alkali vapors," Physical Review Letters, 1967, 18(15): 577-580. 
[2] H. B. Dang, A. C. Maloof, and M. V. Romalis, "Ultrahigh sensitivity magnetic field and magnetization measurements with an atomic magnetometer," Applied Physics Letters, 2010, 97(15): 151110-1-151110-4.

[3] D. Cohen, "Magnetoencephalography: detection of the brain's electrical activity with a superconducting magnetometer," Science, 1972, 175(4022): 664-666.

[4] D. Budker, "Atomic physics-A new spin on magnetometry," Nature, 2003, 422(6932): 574-575.

[5] H. Xia, A. B. A. Baranga, D. Hoffman, and M. V. Romalis, "Magnetoencephalography with an atomic magnetometer," Applied Physics Letters, 2006, 75(21): 211104-1-211104-3.

[6] R. Wyllie, M. Kauer, G. S. Smetana, R. T. Wakai, and T. G. Walker, "Magnetocardiography with a modular spin-exchange relaxation-free atomic magnetometer array," Physics in Medicine and Biology, 2012, 57(9): 2619-2632.

[7] E. Boto, N. Holmes, J. Leggett, G. Roberts, V. K. Shah, S. S. Meyer, et al., "Moving magnetoencephalography towards real-world applications with a wearable system," Nature, 2018, 555(7698): 657-661.

[8] T. Sander, J. Preusser, R. R. Mhaskar, J. Kitching, L. Trahms, and S. Knappe, "Magnetoencephalography with a chip-scale atomic magnetometer," Biomedical Optics Express, 2012, 3(5): 981-990.

[9] A. Borna, T. R. Carter, P. Derego, C. D. James, and P. D. D. Schwindt, "Magnetic source imaging using a pulsed optically pumped magnetometer array," IEEE Transactions on Instrumentation and Measurement, 2018, 68(2): 493-501.

[10]A. Weis, "Optically pumped alkali magnetometers for biomedical applications," Europhysics News, 2012, 43(3): 20-23.

[11] C. Johnson, N. L. Adolphi, K. L. Butler, D. M. Lovato, R. Larson, P. D. D. Schwindt, et al., "Magnetic relaxometry with an atomic magnetometer and SQUID sensors on targeted cancer cells," Journal of Magnetism and Magnetic Materials, 2012, 324(17): 2613-2619.

[12] A. Weis, S. Colombo, V. Dolgovskiy, Z. D. Grujic, V. N. Lebedev, and J. Zhang, "Characterizing and imaging magnetic nanoparticles by optical magnetometry," Journal of Physics Conference Series, 2017, 793(1): 012032-1-012032-4.

[13] G. Bison, N. Castagna, A. Hofer, P. Knowles, J. L. Schenker, M. Kasprzak, et al., "A room temperature 19-channel magnetic field mapping device for cardiac signals," Applied Physics Letters, 2009, 95(17): 173701-1-173701-3.

[14] E. Boto, N. Holmes, J. Leggett, G. Roberts, V. Shah, S. S. Meyer, et al., "Moving magnetoencephalography towards real-world applications with a wearable system," Nature, 2018, 555(7698): 657-661.

[15] C. Johnson, P. D. D. Schwindt, and M. Weisend, "Magnetoencephalography with a two-color pump-probe, fiber-coupled atomic magnetometer," Applied Physics Letters, 2010, 97(24): 413-375.

[16] R. Wyllie, M. Kauer, G. S. Smetana, R. T. Wakai, and T. G. Walker, "Magnetocardiography with a modular spin-exchange relaxation-free atomic magnetometer array," Physics in Medicine and Biology, 2012, 57(9): 2619-2132.

[17] A. Borna, T. R. Carter, J. D. Goldberg, A. P. Colombo, Y. Jau, C. Berry, et al., "A 20-channel magnetoencephalography system based on optically pumped magnetometers," Physics in Medicine and Biology, 2017, 62(23): 8909-8923.

[18] N. Shibata, M. Ohashi, T. Wakabayashi, K. Tsuchiya, S. I. Furukawa, H. Mizuguchi, et al., "Polarization mode coupling and spatial power spectrum of fluctuations along a highly birefringent holey fiber," Journal of Lightwave Technology, 2009, 27(10): 1269-1278.

[19] D. V. Kuksenkov, H. Temkin, and S. Swirhun, "Polarization instability and relative intensity noise in vertical-cavity surface-emitting lasers," Applied Physics Letters, 1995, 67(15): 2141-2143.

[20] K. D. Choquette, R. P. Schneider, K. L. Lear, and R. E. Leibenguth, "Gain-dependent polarization properties of vertical-cavity lasers," IEEE Journal of Selected Topics in Quantum Electronics, 1995, 1(2): 661-666.

[21] J. C. Camparo and R. MacKay, "Spectral mode changes in an alkali rf discharge," Journal of Applied Physics, 2007, 101(5): 53303-153303-6.

[22] A. Dandridge and A. B. Tveten, "Noise reduction in fiber-optic interferometer systems," Applied Optics, 1981, 20(14): 2337-2339.

[23] T. Komljenovic, M. A. Tran, M. Belt, S. Gundavarapu, D. J. Blumenthal, and J. E. Bowers, "Frequency modulated lasers for interferometric optical gyroscopes," Optics Letters, 2016, 41(8): 1773-1776.

[24] G. M. Müller, X. Gu, L. Yang, A. Frank, and K. Bohnert, "Inherent temperature compensation of fiber-optic current sensors employing spun highly birefringent fiber," Optics Express, 2016, 24(10): 11164-11173.

[25] K. Bohnert, P. Gabus, J. Nehring, H. Brändle, and M. G. Brunzel, "Fiber-optic current sensor for electrowinning of metals," Journal of Lightwave Technology, 2007, 25(11): 3602-3609.

[26] M. P. Ledbetter, I. M. Savukov, V. M. Acosta, D. Budker, and M. Romalis, "Spin-exchange-relaxation-free magnetometry with 
Cs vapor," Physical Review A, 2008, 77(3): 033408-1-033408-8.

[27] S. J. Seltzer, "Developments in alkali-metal atomic magnetometry," Ph.D. dissertation, Princeton University, Princeton, New Jersey, USA, 2008.

[28] M. V. Romalis, "Hybrid optical pumping of optically dense alkali-metal vapor without quenching gas," Physical Review Letters, 2010, 105(24): 243001-1-243001-4.

[29] J. C. Fang, T. Wang, W. Quan, H. Yuan, H. Zhang,
Y. Li, et al., "In situ magnetic compensation for potassium spin-exchange relaxation-free magnetometer considering probe beam pumping effect," Review of Scientific Instruments, 2014, 85(6): 63108-1-063108-7.

[30] G. Vasilakis, J. M. Brown, T. W. Kornack, and M. Romalis, "Limits on new long range nuclear spin-dependent forces set with a K-3He comagnetometer," Physical Review Letters, 2009, 103(26): 261801-1-261801-4. 